\title{
Current trends in gene recovery mediated by the CRISPR-Cas system
}

\author{
Hyeon-Ki Jang (1)', Beomjong Song ${ }^{2}$, Gue-Ho Hwang ${ }^{1}$ and Sangsu Bae [1]
}

\begin{abstract}
The CRISPR-Cas system has undoubtedly revolutionized the genome editing field, enabling targeted gene disruption, regulation, and recovery in a guide RNA-specific manner. In this review, we focus on currently available gene recovery strategies that use CRISPR nucleases, particularly for the treatment of genetic disorders. Through the action of DNA repair mechanisms, CRISPR-mediated DNA cleavage at a genomic target can shift the reading frame to correct abnormal frameshifts, whereas DNA cleavage at two sites, which can induce large deletions or inversions, can correct structural abnormalities in DNA. Homology-mediated or homology-independent gene recovery strategies that require donor DNAs have been developed and widely applied to precisely correct mutated sequences in genes of interest. In contrast to the DNA cleavage-mediated gene correction methods listed above, base-editing tools enable base conversion in the absence of donor DNAs. In addition, CRISPR-associated transposases have been harnessed to generate a targeted knockin, and prime editors have been developed to edit tens of nucleotides in cells. Here, we introduce currently developed gene recovery strategies and discuss the pros and cons of each.
\end{abstract}

\section{Introduction}

Human genetic disorders, often associated with severe pathological phenotypes, are caused by genomic aberrations such as gene mutations and chromosomal abnormalities. Therefore, a reliable therapeutic method for gene recovery would be quite valuable. Previously, exogenous delivery of therapeutic normal genes via viral vehicles, such as adenoviruses, adeno-associated viruses (AAVs), and lentiviruses, has been tried as a means of providing the normal function of the inactivated/disrupted gene ${ }^{1}$. Although such gene therapy methods have produced successful therapeutic results, this general approach has potential limitations. For example, the exogenous gene is constitutively expressed, unaffected by the native chromatin structure of the endogenous locus, at a level that

Correspondence: Sangsu Bae (sangsubae@hanyang.ac.kr)

${ }^{1}$ Department of Chemistry and Research Institute for Convergence of Basic Sciences, Hanyang University, Seoul 04763, South Korea

${ }^{2}$ International Research Center for Neurointelligence (WPI-IRCN), The University of Tokyo, Hongo, Bunkyo-ku, Tokyo 113-0033, Japan

These authors contributed equally: Hyeon-Ki Jang, Beomjong Song differs from that of the endogenous gene ${ }^{2}$. Furthermore, the mutated endogenous gene, which is malfunctional and potentially cytotoxic, might still be transcribed.

Precise correction of the endogenous gene of interest is a strongly desirable alternative for gene recovery. Programmable nucleases, which include zinc-finger nucleases $(\mathrm{ZFNs})^{3}$, transcription activator-like effector nucleases $(\mathrm{TALENs})^{4}$, and clustered regularly interspaced short palindromic repeat (CRISPR)-CRISPR-associated (Cas) endonucleases $^{5-7}$, enable target-specific DNA cleavage and gene editing. CRISPR-mediated gene-editing technologies are now overwhelmingly the method of choice because of their ease of handling and low cost. Since CRISPR nucleases were first harnessed for generating sitespecific DNA cleavage in the human genome, new CRISPR-based gene-editing tools, including base-editing technologies, have been developed. The ability to correct endogenous genes in a targeted and predictable manner using such tools has undoubtedly revolutionized genebased drug development as well as basic research. In this review, we introduce current trends in CRISPR-mediated 
gene correction and rescue strategies and describe the pros and cons of each tool.

\section{DNA repair pathways induced by CRISPR- mediated DNA cleavage in eukaryotic cells}

The type II CRISPR-Cas9 and type V CRISPR-Cas12a (also known as Cpf1) endonucleases are targeted to specific genomic sites by associated guide $\mathrm{RNAs}^{8,9}$ and can be used to generate site-specific DNA cleavage in various cell types and organisms, including humans. Typically, researchers use one piece of single guide RNA (sgRNA), which includes a spacer region complementary to the target DNA and a region that binds to the endonuclease. The target DNA sequence recognized by the guide RNA must be associated with a nuclease-specific protospacer adjacent motif (PAM), which is recognized directly by the endonuclease.

The chromosomal double-strand breaks (DSBs) produced by these nucleases are typically repaired by the cell's own repair processes, such as the non-homologous end joining (NHEJ) pathway, the homology-directed repair (HDR) pathway, or an alternative KU-independent process such as the microhomology-mediated end joining (MMEJ) pathway ${ }^{10}$ (Fig. 1). DSBs are ligated without a homologous template during the NHEJ process, which frequently leads to small nucleotide insertions and deletions (indels) at the cleavage site. In the presence of a donor DNA template, HDR precisely rejoins the DSB ends based on the donor DNA sequence, which results in precise gene corrections or knockins. The MMEJ pathway is an alternative NHEJ pathway that involves annealing between identical microhomologous sequences ( $>2 \mathrm{bp}$ ) flanking the DSB. Hence, MMEJ causes sequence-dependent deletions according to the microhomologous sequences that flank the cleavage site. On the basis of these various repair pathways, researchers have established precise endogenous gene recovery strategies in human cells for treating different genetic diseases.

\section{Gene recovery strategies in the absence of donor DNA}

Frameshift- and deletion-mediated gene recovery involving one guide RNA

NHEJ, a dominant repair pathway in mammalian cells that is active throughout the cell cycle ${ }^{10}$, is typically used for gene disruption or knockout via induction of indels $^{11,12}$. Alternatively, however, NHEJ-mediated indels can be effectively used for genetic disease treatment if they induce a desired frameshift or delete a point mutation (Fig. 2a). For example, in Duchenne muscular dystrophy (DMD) models, premature stop codons induced by deletion of exon 44 were corrected by the introduction of Cas9-mediated frameshifting indels at a nearby location ${ }^{13,14}$. Additionally, point mutations that lead to aberrant splicing in the $D M D$ gene or in the Hemoglobin $B(H B B)$ gene, which cause $\beta$-thalassemia, were removed by the introduction of Cas9- or Cas12amediated indels ${ }^{15,16}$.

Similar to the NHEJ-mediated gene recovery strategy, MMEJ-mediated deletions can also be used to remove disease-causing mutations (Fig. 2a). An 8-bp duplication in exon 1 of the TCAP gene that causes limb-girdle muscular dystrophy type 2 G (LGMD2G) was deleted precisely in patient-derived induced pluripotent stem cells (iPSCs) and myoblasts differentiated from the iPS cells via the MMEJ pathway ${ }^{17}$. Likewise, MMEJ was utilized to remove a 16-bp microduplication in exon 15 of the HPS1 gene in B lymphocytes that causes Hermansky-Pudlak syndrome type-1 (HPS1).

In addition, several programs, such as Microhomology predictor $^{18}$, inDelPhi ${ }^{19}$, DeepSpCas $9^{20}$, and DeepCpf $1^{21}$, have been developed to predict gene-editing efficiencies and/or editing outcomes after CRISPR treatment. These

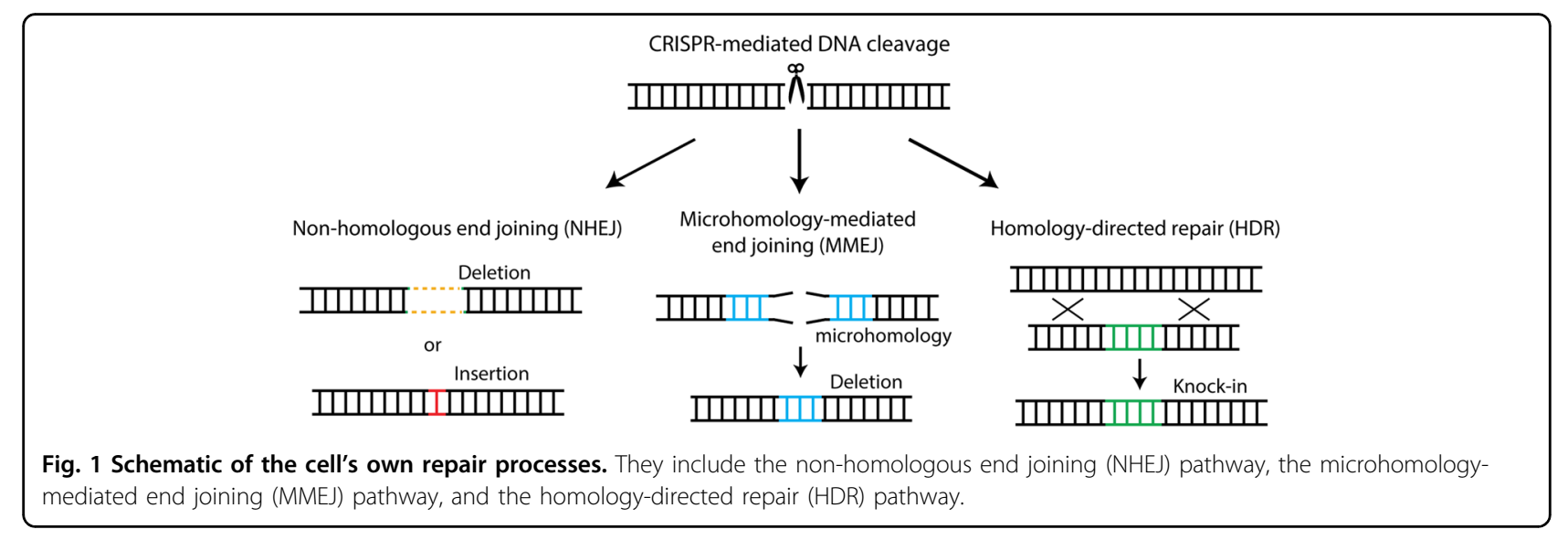




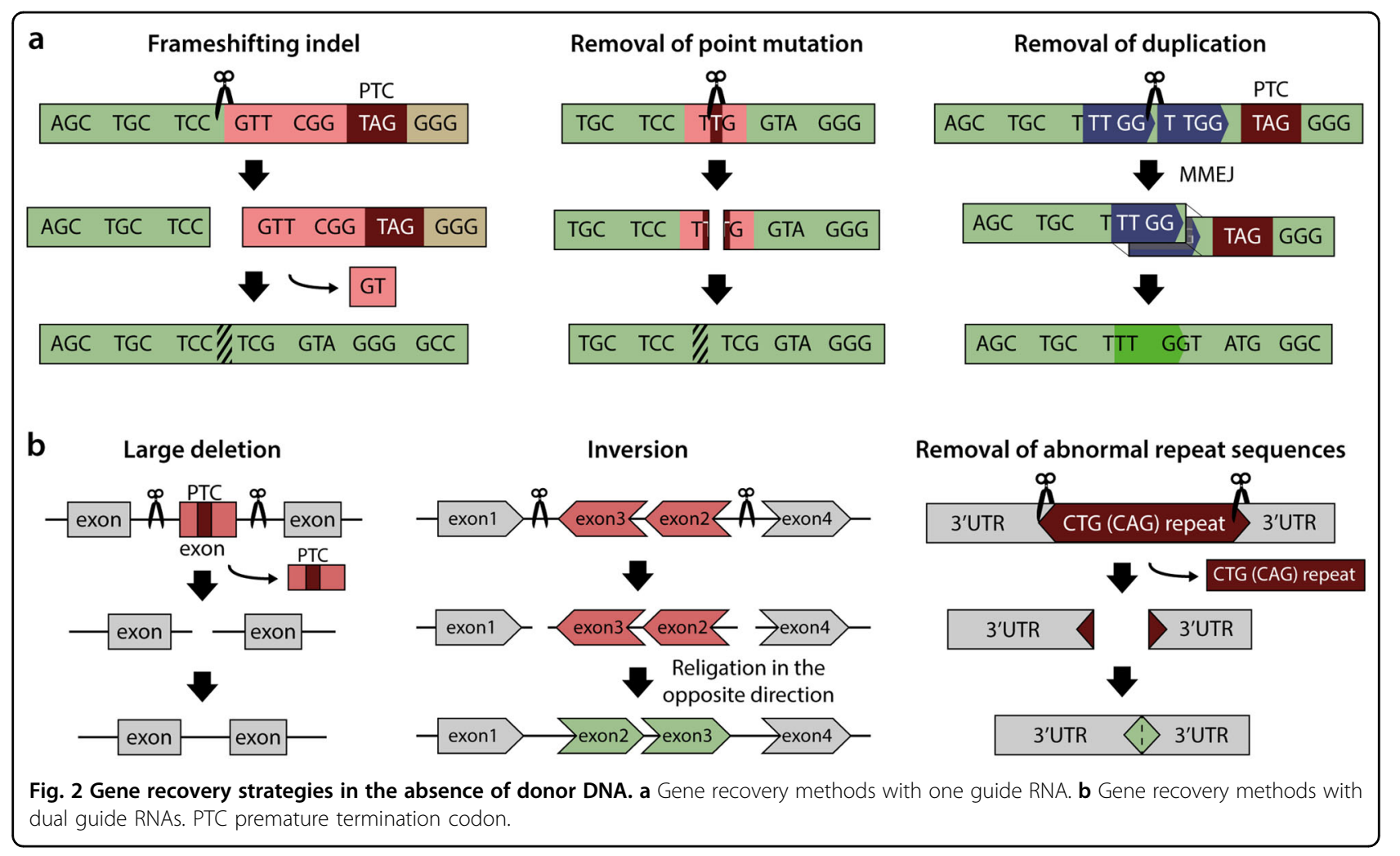

resources should accelerate the use of frameshift- and small deletion-mediated gene recovery strategies.

\section{Large deletion- or inversion-mediated gene recovery involving two guide RNAs}

CRISPR nuclease target sites can be changed simply by altering the sgRNA sequence; multiple DNA cleavages are easily obtained by using two or more sgRNAs simultaneously. Therefore, researchers can generate a large deletion or inversion of a gene of interest by using dual sgRNAs (Fig. 2b). Because CRISPR-mediated DNA cleavage is typically accompanied by indel formation at target sites, introns may be more suitable than exons as target sites in such approaches. For example, the abnormal CTG repeat in the $3^{\prime}$ untranslated region of the $D M P K$ gene that results in myotonic dystrophy type- 1 was successfully excised in patient-derived iPSCs and muscle cells by treatment with Cas9 and dual $\operatorname{sgRNAs}^{22,23}$. Additionally, mutationcarrying exons were excised by using dual sgRNAs in myoblasts derived from a DMD mouse model and in keratinocytes derived from patients with recessive dystrophic epidermolysis bullosa (RDEB); this approach restored gene function $^{24,25}$. Cas9 with dual sgRNAs can induce not only large deletions but also inversions, which occur when the excised gene fragment is religated in the opposite direction at the same locus. A large inversion in the FVIII gene associated with hemophilia A was reoriented by using Cas9 with dual sgRNAs in hemophilia A patient-derived iPSCs ${ }^{26}$.

\section{Gene recovery strategies involving donor DNAs Precise HDR-mediated gene correction}

HDR-mediated gene correction is the most popular strategy for gene recovery because the genetic defect is corrected to exactly match the DNA donor template. To date, HDR-mediated gene correction has been widely harnessed to treat various genetic diseases, including sickle cell disease $\mathrm{e}^{27-29}$, B-thalassemia ${ }^{30-34}$, hemophilia $\mathrm{A} / \mathrm{B}^{35-38}$, and $\mathrm{DMD}^{14,39,40}$, in cells or organisms. The DNA donors for HDR have been provided in various forms, including single-stranded DNA oligonucleotides (ssODNs), DNA plasmids or viral vectors, as discussed below $^{41}$ (Fig. 3a).

For correction of one or a few mutations, an ssODN donor template is preferable because of its low cost and the relatively high associated editing efficacy. The relatively small size of ssODNs $(90 \sim 200 \mathrm{nt})$ is advantageous for the synthesis and delivery of the donor template. Furthermore, ssODNs show a low level of chromosomal integration compared to double-stranded DNA oligonucleotides $(\mathrm{dsODNs})^{42}$. A dominant cataract disorder in mice was the first genetic disease for which the ssODN strategy was used to correct the causative mutation, a 1-bp deletion in exon 3 of the Crygc gene ${ }^{43}$ that leads to the production of truncated $\gamma \mathrm{C}$-crystallin ${ }^{44}$. Other diseases associated with single base substitutions, such as DMD (DMD gene $)^{39,40}$, achondroplasia (FGFR3 gene $)^{45}$, Alzheimer's disease (PSEN2 gene $)^{46}$, retinitis pigmentosa 


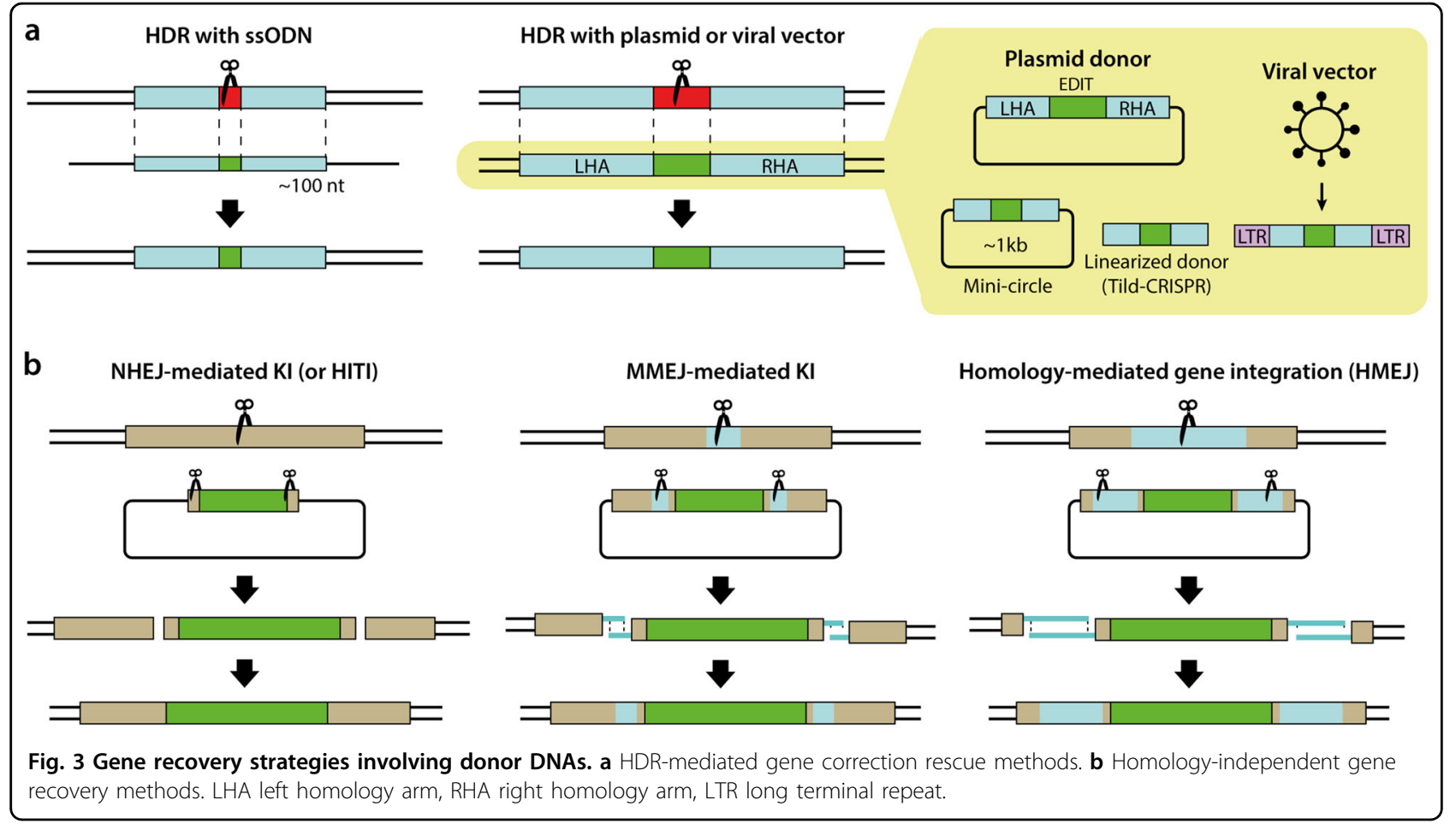

$(P d e 6 b \text { gene })^{47}$, sickle cell disease $(H B B \text { gene })^{48}$, and hemophilia B ( $H B B$ gene $)^{37}$, have also been targeted with ssODN-templated gene correction. Additionally, a 4-bp deletion in the $H B B$ gene (causing $\beta$-thalassemia) ${ }^{32,33}$ and a 4-bp insertion in the FANCF gene (causing Fanconi anemia $^{49}$ were corrected exactly by the ssODNtemplated HDR strategy.

The ssODN strategy is convenient for gene correction at the cellular level but is not easily applied for in vivo corrections in organisms such as mice due to the lack of a reliable delivery method. For example, ssODNs cannot be carried by viral vehicles, in contrast with DNA plasmids. Hence, donor templates for in vivo applications are typically prepared as plasmids carrying the desired sequence flanked by long homology arms ( $200 \mathrm{bp})$ (Fig. 3b). The donor plasmid template resembles an endogenous template (i.e., the opposite allele) in the homologous recombination process. HDR-mediated genome editing with a plasmid donor can precisely replace a long stretch of nucleotides or insert large constructs, such as a sequence encoding a tagged protein, at a specific locus. Cystic fibrosis was the first genetic disease for which HDRmediated gene correction with a donor plasmid was performed in patient-derived intestinal stem cells carrying a homozygous 3-bp deletion in exon 11 of the CFTR gene $^{50}$. The donor plasmid contained a puromycin resistance gene in addition to the wild-type CFTR sequence for effective selection of knockin cells. Indeed, the availability of selection markers, including drug resistance genes or genes encoding fluorescent proteins, is one of the benefits of using a plasmid donor versus an ssODN donor. By using HDR with a plasmid carrying donor DNA, various mutation patterns have been corrected, including smallsized mutations in the $H B B$ gene (associated with

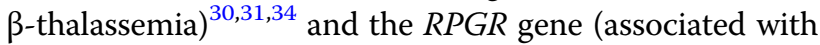
retinitis pigmentosa) ${ }^{51}$, as well as large-sized mutations corrected by the precise knockin of a large DNA fragment from the FVIII gene (associated with hemophilia A) ${ }^{38}$ and from the $D M D$ gene (associated with DMD) ${ }^{13}$.

However, the efficiency of plasmid-templated HDR is typically lower than that of ssODN-templated HDR. To enhance the editing efficiency of plasmid-templated HDR, some modifications have been adopted. A minicircle plasmid $(\sim 1 \mathrm{~kb})$, the size of which was minimized by removal of the bacterial backbone sequence, was utilized as a donor template to increase transfection efficiency ${ }^{52}$. Additionally, linearized templates that were prepared by PCR amplification or restriction enzyme-mediated digestion were associated with increased editing efficiencies in various cell types; this method was termed targeted integration with linearized dsDNA-CRISPR (Tild-CRISPR) $)^{53}$.

To maximize donor plasmid delivery into the cell nucleus, recent studies used viral vectors, including an integrase-defective lentiviral vector, adenoviral vector, or adeno-associated viral vector, as the donor template. Genetic mutations associated with $\mathrm{SCD}^{27-29}$, hemophilia $\mathrm{B}^{35,36}$, or $\mathrm{RDEB}^{54}$ were corrected by Cas9-mediated HDR using a viral vector donor. Delivery of an AAV6 donor 
along with Cas9 RNPs to SCD patient-derived hematopoietic stem and progenitor cells (HSPCs) resulted in successful targeted gene correction at 19\% efficiency ${ }^{27}$. The combination of Cas9 RNPs and an AAV6 donor template has been reported to be a powerful tool for gene correction in HSPCs, T cells, and iPSCs, resulting in precise editing efficiencies of $\sim 60 \%{ }^{29,55,56}$. Notably, AAV donors can be used as an alternative to plasmid donors, especially in some cell types that show poor HDR editing efficiency or cytotoxicity when transfected with plasmids.

\section{Homology-independent gene recovery}

Despite the precision of HDR-mediated gene correction, it is not the method of choice in all circumstances. This method frequently shows low editing efficacy, and its utility is limited in non-dividing or fully differentiated cells, because HDR is active only in the late $S$ and G2 phases of the cell cycle ${ }^{57}$. In contrast, gene expression cassettes can be integrated via the NHEJ-mediated knockin method regardless of the cell cycle phase ${ }^{58}$ (Fig. 3b). The representative example of this method is homology-independent targeted integration (HITI $)^{59}$. HITI employs donor plasmids that lack homology arms but include Cas9 cleavage sites flanking the donor sequence. Therefore, Cas9 nucleases cut both the genomic target sequence and the donor plasmid, after which the cleaved donor DNA can be incorporated into the target gene. One remarkable property of HITI is its high accuracy even in fully differentiated cells such as neurons ${ }^{60}$, which can be achieved by repeating Cas9mediated cleavage until the donor DNA is inserted in the desired orientation ${ }^{59}$. This property of HITI makes it a promising method for curing genetic diseases by gene replacement. Royal College of Surgeons (RCS) rats, an animal model of retinitis pigmentosa caused by deletion in the Mertk gene, have morphological changes in the degenerating photoreceptor outer nuclear layer $(\mathrm{ONL})^{61}$. Injection of HITI-AAV vectors in the subretinal space in the eyes of RCS rats significantly increased Mertk mRNA levels and preserved ONL thickness ${ }^{59}$. Consistent with this, the MERTK protein was observed in the eyes, and electroretinography tests showed improved eye function ${ }^{59}$.

Similar to NHEJ, MMEJ can also mediate knockin of a large gene construct. In a technique known as CRISPITCh, CRISPR nucleases are used to cleave both genomic and donor DNA at sites with microhomology, resulting in precise integration into the target chromosome $^{62}$ (Fig. 3b). CRIS-PITCh requires three sgRNAs, and the CRIS-PITCh vector must include two different sgRNA target sites. A recent study showed the potential for CRISPITCh in gene replacement therapy. Hydrodynamic injection of Cas9-expressing and Fah-MMEJ constructs into $\mathrm{Fah}^{-/-}$mouse livers, which are a model of hereditary tyrosinemia type I (HTI) caused by a deficiency in fumarylacetoacetate hydrolase due to mutation of the Fah gene, resulted in correction of the Fah gene in hepatocytes, which alleviated symptoms such as body weight loss and liver damage as well as prolonged the lives of the Fah-corrected mice ${ }^{63}$.

To maximize the editing efficacy relative to that seen with the HDR-, NHEJ-, and MMEJ-mediated knockin strategies described above, a new combination strategy using long homology arms ( $~ 800 \mathrm{bp})$ was developed, referred to as homology-mediated gene integration $(\mathrm{HMEJ})^{64}$ (Fig. 3b). In this technique, the HMEJ construct contains homology arms similar to those used for HDR as well as CRISPR targets flanking the donor DNA similar to those used for NHEJ, enabling HMEJ to be mediated by either NHEJ or HDR depending on the cell type. In nondividing cells such as astrocytes and neurons, HMEJ resulted in a gene-editing efficiency that was comparable with that of NHEJ- and MMEJ-based methods. Interestingly, an HDR inhibitor (caffeine) decreased HMEJmediated gene knockin in mouse embryonic stem (ES) cells but not in neurons, whereas an NHEJ inhibitor (Scr7 or Nu7026) decreased the HMEJ-mediated gene-editing efficiency in neurons but not in mouse ES cells ${ }^{64}$. This dual nature of HMEJ represents a ray of hope in gene therapy. Indeed, HMEJ was effective for treating HTI. Hydrodynamic injection of HMEJ constructs into $\mathrm{Fah}^{-/-}$ mice resulted in greater proliferation of normal hepatocytes than the MMEJ strategy ${ }^{65}$.

\section{Gene recovery by base editors without DNA DSB generation}

Mutation of a single nucleotide in a gene, which can induce an amino acid substitution in the encoded protein (missense mutation) or truncation of the protein (nonsense mutation), is the main cause of genetic diseases $\left(>58 \%\right.$ of the entries in the ClinVar database) ${ }^{66}$. Although the single mutated nucleotide can be precisely repaired through HDR, the low editing efficiency and the restriction to non-dividing cells of this method obstruct its therapeutic application. In addition, recent studies have warned that DNA DSBs can lead to a p53-mediated DNA damage response $\mathrm{e}^{67,68}$ and can frequently cause unexpected large deletions ${ }^{69,70}$. Therefore, an alternative gene correction method that does not generate DNA DSBs is required. CRISPR-based base-editing tools, including cytosine and adenine base editors (CBEs and ABEs), have recently been developed for highly efficient single nucleotide correction, which occurs without donor DNA but does not generate DNA DSBs ${ }^{71,72}$.

\section{Cytosine base editors (CBEs)}

The optimized forms of CBEs (BE3 and TARGET-AID) are composed of three proteins: a partially inactive Cas9 
a Cytosine Base Editor (CBE)

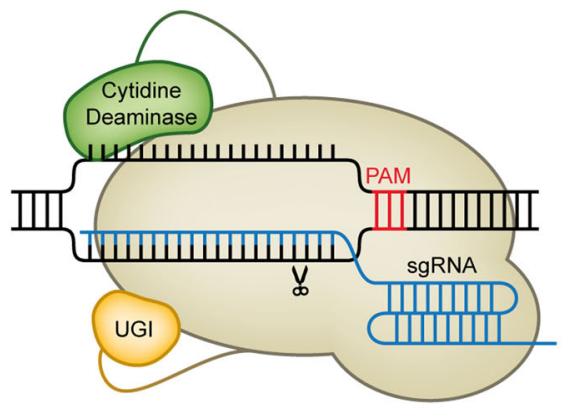

b CRISPR-associated transposase (CAST)

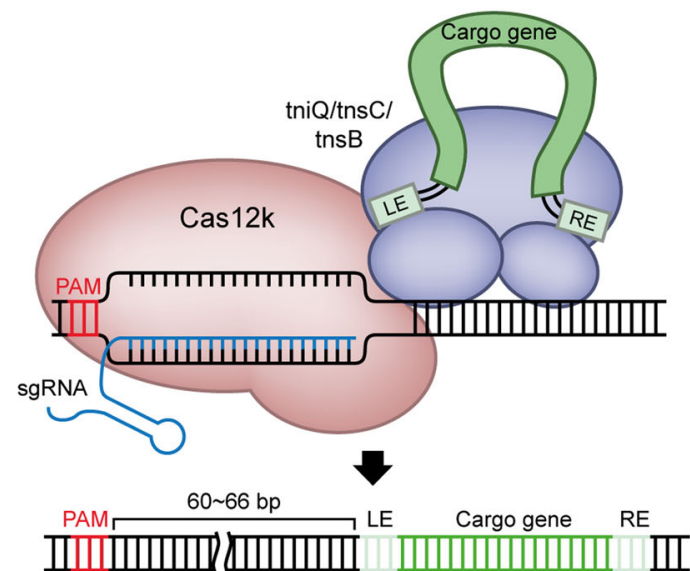

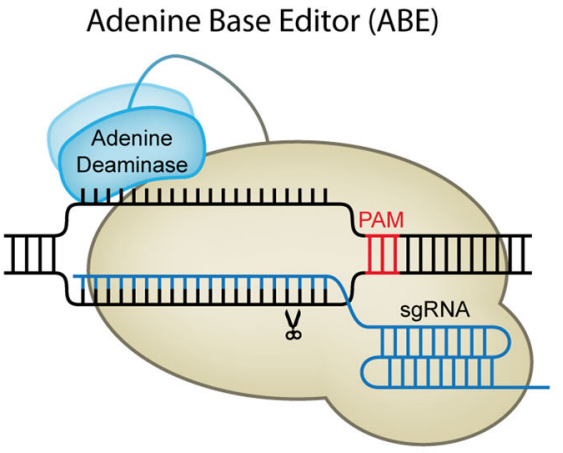

Type I transposon-associated CRISPR-Cas (INTEGRATE)

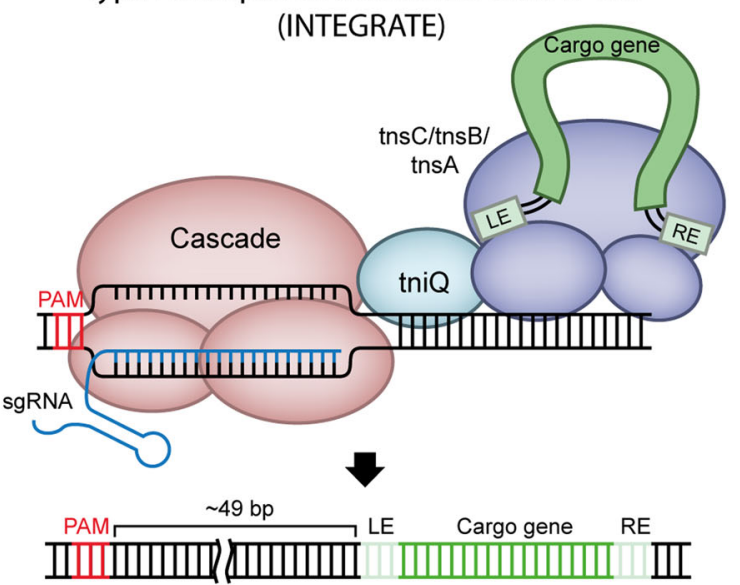

c Prime Editor (PE)

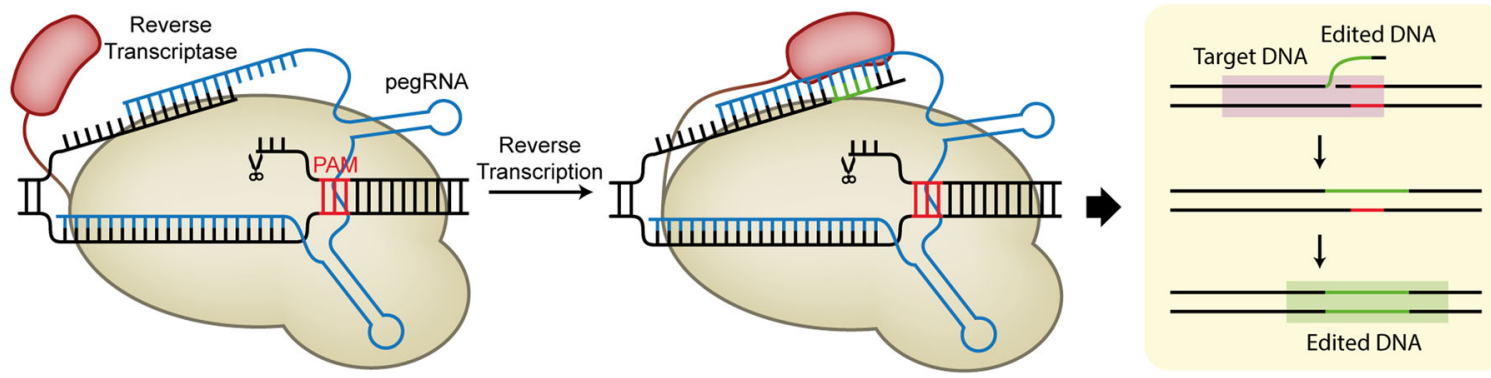

Fig. 4 Gene recovery strategies in the absence of DNA DSB generation. a Schematic of a cytosine base editor (CBE), an adenine base editor (ABE). $\mathbf{b}$ Schematic of the insertion of transposable elements by guide RNA-assisted targeting (INTEGRATE) with a type I transposon-associated CRISPR-Cas system and of RNA-guided DNA insertion with a CRISPR-associated transposase (CAST). LE transposon left end, RE transposon right end. c Schematic of a prime editor (PE) and its working mechanism. Reverse transcriptases in PEs copy the information in the pegRNAs into DNA target sites.

variant that exhibits nickase activity (nCas9) fused to a cytidine deaminase such as rAPOBEC1 or pmCDA1 and a uracil DNA glycosylase inhibitor (UGI) ${ }^{71,73}$ (Fig. 4). The CBE initially replaces a cytosine in the nontarget strand with uracil, after which the guanine in the target strand that was previously paired with cytosine is replaced with adenine by the cell's repair mechanism. Finally, the uracil is replaced with thymine, thereby generating a $\mathrm{T}-\mathrm{A}$ pair in place of the previous $\mathrm{C}-\mathrm{G}$ pair $^{71}$. UGI prevents the base excision repair process that removes uracil from the nontarget strand, enhancing the C-to- $\mathrm{T}$ conversion efficiency.

Subsequent studies led to the development of several $\mathrm{CBE}$ variants with improved editing efficiency. The 
editing window of $\mathrm{BE} 3$ was narrowed by altering the sequences linking nCas9 to the cytidine deaminase ${ }^{74}$ or by APOBEC1 mutation $^{75}$, and BE3 expression was enhanced in mammalian cells by optimizing the nCas9-encoding sequences $^{76}$. Adding a second UGI on BE3 (BE4) decreased the formation of undesired byproducts of editing ${ }^{77}$. BE4 was further improved by adopting nuclear localization signal (NLS) sequences and optimized codons (BE4max) and by employing APOBEC homologs $(\text { AncBE4max })^{78}$.

CBEs show great promise for the treatment of genetic diseases because many preliminary studies have shown tangible results. For example, nucleofection of astrocytes with $\mathrm{BE} 3$ constructs converted the apolipoprotein $\mathrm{E}$ gene 4. (APOE4), the most common genetic risk factor for lateonset Alzheimer's disease, to $A P E 3 r$, a less risky form ${ }^{71}$. The p53 gene mutation, which causes many types of cancers, was corrected by BE3 in human breast cancer cells $(\mathrm{HCC1954})^{71}$. A nonsense mutation in the superoxide dismutase 1 (SOD1) gene was induced by BE3 in amyotrophic lateral sclerosis (ALS) model mice, prolonging survival ${ }^{79}$. The $H B B$ gene mutation that causes $\beta$-thalassemia was corrected by BE3 or its variant YEEBE3 in skin fibroblasts, human embryos, and blastomeres $^{80}$. The FBN1 mutation, a cause of Marfan syndrome $^{81}$, was repaired in human embryos using YE1-BE3 or YEE-BE3 ${ }^{82}$.

A successful CRISPR-based gene correction strategy requires that an appropriate PAM be located near the mutation of interest. The most commonly used Cas9 nuclease, SpCas9 from Streptococcus pyogenes, recognizes a $5^{\prime}$-NGG-3' PAM downstream of the target, but such motifs are not always present. This limitation can be overcome by swapping the Cas9 nickase with other nickases preferring different PAM sequences. nSaKKHBE3, which adopts mutated SaCas9 derived from Staphylococcus aureus, recognizes alternative PAM sequences $\left(5^{\prime}\right.$-NNNRRT- $\left.3^{\prime}\right)$ and was used to correct the Pah gene mutation in the hepatocytes of phenylketonuria model mice ${ }^{83}$.

\section{Adenine base editors (ABEs)}

Analogous to CBEs, ABEs replace adenines in the nontarget strand with inosines, generating $\mathrm{I}-\mathrm{T}$ pairs. Because inosine preferentially base pairs with cytosine over thymine, DNA repair machinery converts the I-T pairs to I-C pairs and finally to G-C pairs ${ }^{72}$. ABEs differ from $\mathrm{CBEs}$ in that they contain deoxyadenosine deaminase instead of cytidine deaminase (Fig. 4). Because a natural deoxyadenosine deaminase is not known to exist, directed evolution for protein engineering was used to convert a transfer RNA adenosine deaminase, TadA, to a DNA deoxyadenosine deaminase, referred to as TadA*, which was then fused to a nCas9 to create ABE7.10 ${ }^{72}$.
This initially optimized $\mathrm{ABE}$ was further improved by optimizing codons and adding an NLS sequence (ABE$\max )^{78}$. Recently, various ABEmax variants, based on different Cas9 variants, were developed (VRQR-ABEmax, VRER-ABEmax, xABEmax, NG-ABEmax, SaABEmax, and SaKKH-ABEmax); these versions expand the target sites of $\mathrm{ABEs}^{84}$.

Similar to the situation with CBEs, there have been many efforts to apply ABEs to gene therapy. For example, the expression of the $H B G 1$ and $H B G 2$ genes in adults is thought to alleviate the symptoms of $\beta$-globin-related blood diseases, and ABE7.10 and ABEmax were successfully used to induce the desired mutation in the $H B G 1$ and HBG2 promoters in HEK293T cells ${ }^{72,78}$. The mutation in the HFE gene causing hereditary hemochromatosis was corrected by ABE7.10 in an immortalized lymphoblastoid cell line ${ }^{72}$. In addition, by converting a premature stop codon in $D M D$ to a Glu codon, ABE7.10 restored dystrophin expression in myofibers of DMD model mice ${ }^{85}$. In HTI model mice, targeting RA6.3 (an improved ABE6.3 variant) to the Fah gene corrected the HTIassociated mutation and alleviated disease symptoms ${ }^{86}$. In another example, a mutation in the COL7A1 gene causing recessive $\mathrm{RDEB}^{87,88}$ was corrected by $\mathrm{ABEmax}$ in primary fibroblasts and iPSCs ${ }^{89}$. Furthermore, CjABE, an ABE that contains catalytically impaired Cas9 from Campylobacter jejuni, was used to correct a brain tumor-associated mutation in the TRET gene in primary glioblastomas ${ }^{90}$. Finally, CRISPR-pass is a general ABE-based method of inducing premature stop codon read-through. When used in fibroblasts from xeroderma pigmentosum complement group C (XPC) patients, this technique led to readthrough of the disease-associated premature stop codon, thereby generating functional protein ${ }^{91}$.

\section{New gene recovery strategies: CRISPR-associated transposase and prime editors}

Recently, it was reported that several Tn7-like transposons are associated with CRISPR-Cas systems ${ }^{92,93}$; this finding has led to the development of new gene-editing tools (Fig. 4). One approach is to use a CRISPR-associated transposase from the cyanobacterium Scytonema hofmanni (ShCAST); this transposase is made up of Tn7-like transposase subunits and a type V Cas $12 \mathrm{k}^{94}$. The ShCAST complex is recruited to the target site in an RNA-guided manner due to the type $\mathrm{V}$ Cas12k and unidirectionally inserts the cargo genes of the donor plasmid into the target site via its transposase subunits. The other tool is insertion of transposable elements by guide RNA-assisted targeting (INTEGRATE) based on a type I transposonassociated CRISPR-Cas system ${ }^{95}$. INTEGRATE consists of a CRISPR-associated complex for antiviral defense (Cascade) complex (Cas6, Cas7, and Cas8), which directs the editing machinery to the target site, and Tn7-like 
Table 1 Current gene correction treatments for many genetic diseases.

\begin{tabular}{|c|c|c|c|}
\hline Disease & Target gene & Type of mutation & Gene recovery strategy \\
\hline Achondroplasia & Fgfr3 & c.1120 G > A (p.G374R) & $\mathrm{HDR}(\mathrm{ssODN})^{45}$ \\
\hline \multirow[t]{2}{*}{ Alzheimer's disease (AD) } & PSEN2 & c.422 A > T (p.N141l) & $\mathrm{HDR}(\mathrm{ssODN})^{46}$ \\
\hline & $A P O E$ & ApoE4 Arg158 & $\mathrm{CBE}^{71}$ \\
\hline Amyotrophic lateral sclerosis (ALS) & SOD1 & c.281 G > C (G94A) (G93A transgenic mouse) & $\mathrm{CBE}(\mathrm{KO} \text { of mutant } \mathrm{SOD} 1)^{79}$ \\
\hline \multirow[t]{5}{*}{$\beta$-thalassemia } & \multirow[t]{5}{*}{$H B B$} & $\begin{array}{l}\text { c.93-21 G > A or c.316-197 C > T (inducing an } \\
\text { aberrant splice) }\end{array}$ & Indels to remove a point mutation ${ }^{16}$ \\
\hline & & \multirow[t]{2}{*}{ c.126_129delCTTT (CD 41/42 (-CTTT)) } & $\operatorname{HDR}(\mathrm{ssODN})^{32,33}$ \\
\hline & & & HDR (donor plasmid) $)^{30,34}$ \\
\hline & & c.654 C > T & HDR (donor plasmid) $)^{31}$ \\
\hline & & g. $-28 \mathrm{~A}>\mathrm{G}$ & $\mathrm{CBE}^{80}$ \\
\hline$\beta$-globin-related disease & $H B G 1$ & g. $-175 T$ or g. $-198 \mathrm{~T}^{\mathrm{a}}$ & $\mathrm{ABE}^{72,78}$ \\
\hline Cancer & TP53 & c.488 A > G (p.Y163C) & $\mathrm{CBE}^{71}$ \\
\hline Cataract & Cryge & c.461delG (at exon 3) & HDR (ssODN or w/o donor) ${ }^{43 b}$ \\
\hline Cystic fibrosis & CFTR & c.1521_1523delCTT (p.F508del) & HDR (donor plasmid) ${ }^{50}$ \\
\hline $\begin{array}{l}\text { Congenital disorder of glycosylation (CDG) } \\
\text { type- } 1 \mathrm{f}\end{array}$ & MPDU1 & c.356 T > C (p.L119P) & $\mathrm{CBE}^{78}$ \\
\hline Chronic pain & Scn9a & c.689-1 Ca & $\mathrm{CBE}^{78}$ \\
\hline \multirow[t]{10}{*}{ Duchenne muscular dystrophy (DMD) } & \multirow[t]{10}{*}{$D M D$} & \multirow[t]{3}{*}{ c.2983 C > T (at exon 23, p.Q995X, mdx mouse) } & Large deletion using dual sgRNAs ${ }^{24}$ \\
\hline & & & HDR $(s s O D N)^{39,40}$ \\
\hline & & & HDR (Adv donor) $)^{39}$ \\
\hline & & \multirow{3}{*}{$\begin{array}{l}\text { Deletion of exon } 44 \text { introducing a PTC into } \\
\text { exon } 45\end{array}$} & Frame-fitting indels ${ }^{13,14}$ \\
\hline & & & Indels for skipping exon $45^{13,14}$ \\
\hline & & & HDR (donor plasmid) ${ }^{14}$ \\
\hline & & $\begin{array}{l}\text { Gross deletion of exons } 48-50 \text { introducing a PTC } \\
\text { into exon } 51\end{array}$ & $\begin{array}{l}\text { Indels to disrupt a splice acceptor at } \\
\text { exon } 51 \text { for skipping exon } 51^{15}\end{array}$ \\
\hline & & $\begin{array}{l}\text { c.6913-4037 T > G (a cryptic splice acceptor at } \\
\text { intron 47) }\end{array}$ & $\begin{array}{l}\text { Indels to remove the cryptic splice } \\
\text { acceptor }^{15}\end{array}$ \\
\hline & & Gross duplication of exons 55-59 & Large deletion using dual sgRNAs ${ }^{15}$ \\
\hline & & c.2611 C > T (p.Q871X) & $\mathrm{ABE}^{85}$ \\
\hline Epidermolysis bullosa simplex (EBS) & KRT14 & c.1231 G>A (at exon 6) & HDR (donor plasmid) ${ }^{52}$ \\
\hline Fanconi anemia & FANCF & c.828InsTAAA & $\mathrm{HDR}(\mathrm{ssODN})^{49}$ \\
\hline \multirow[t]{2}{*}{ Hemophilia A (HA) } & \multirow[t]{2}{*}{ FVIII } & $\begin{array}{l}\text { Gross chromosomal inversion of } 140-\mathrm{kbp} \text { or } \\
600-\mathrm{kbp} \text { involving introns } 1 \text { and } 22\end{array}$ & Large inversion using dual sgRNAs ${ }^{26}$ \\
\hline & & $\begin{array}{l}\text { Gross deletion of } 94,172 \mathrm{bp} \text { from exon } 8 \text { to } \\
\text { intron } 22\end{array}$ & HDR (donor plasmid) $)^{38}$ \\
\hline \multirow[t]{4}{*}{ Hemophilia B (HB) } & \multirow[t]{4}{*}{ FIX } & c.1111 T> G (p.Y371D, mouse) & HDR (ssODN or donor plasmid) ${ }^{37}$ \\
\hline & & c.1477 G > A (p.Q418G, dog) & HDR (AAV donor) ${ }^{35}$ \\
\hline & & & HDR (Adv donor) ${ }^{35}$ \\
\hline & & c.1136 G > A (p.R379Q) (R333Q transgenic mouse) & HDR (Adv donor) $)^{36}$ \\
\hline Hereditary tyrosinemia type I (HTI) & Fah & & MMEJ-mediated $\mathrm{KI}^{63}$ \\
\hline
\end{tabular}


Table 1 continued

\begin{tabular}{|c|c|c|c|}
\hline Disease & Target gene & Type of mutation & Gene recovery strategy \\
\hline & & $\begin{array}{l}\text { Insertion of a neomycin selection cassette at exon } \\
5 \text { (Fah }{ }^{\Delta e x o n 5} \text { mouse) }\end{array}$ & HMEJ-mediated $\mathrm{K}^{65}$ \\
\hline & & c.706 G > A (exon 8 skipping, Fah5981SB mouse) & $A B E^{86}$ \\
\hline Hermansky-Pudlak syndrome & HPS1 & c.1472_1487dup (16-bp duplication) & MMEJ J 17,19 \\
\hline Hereditary hemochromatosis ( $\mathrm{HHC}$ ) & HFE & $c .845$ G > A (p.C282Y) & $\mathrm{ABE}^{72}$ \\
\hline Limb-girdle muscular dystrophy (LGMD) & TCAP & c.26_33dup (8-bp duplication) & MMEJ' ${ }^{17}$ \\
\hline Marfan syndrome & FBN1 & c.7498 T > C & $\mathrm{CBE}^{82}$ \\
\hline Menkes disease & ATP7A & c.6913_6917dupCTTAT & MMEJ $^{19}$ \\
\hline Myotonic dystrophy type-1 (DM1) & DMPK & CTG repeat expansion in the $3^{\prime} U T R$ & $\begin{array}{l}\text { Large deletion using dual } \\
\text { sgRNAs }^{22,23}\end{array}$ \\
\hline Phenylketonuria (PKU) & Pah & c.835 T > C (p.F263S) & $\mathrm{CBE}^{83}$ \\
\hline \multirow[t]{3}{*}{ Retinitis pigmentosa } & Pde6b & c.1041 C > A (p.Y347X) & $\mathrm{HDR}(\mathrm{ssODN})^{47}$ \\
\hline & $R P G R$ & c.1685_1686del (at exon 14) & HDR (donor plasmid) ${ }^{51}$ \\
\hline & Mertk & Gross deletion of $1.9 \mathrm{kbp}$ from intron 1 to exon 2 & NHEJ-mediated KI $(H I T I)^{59}$ \\
\hline \multirow{3}{*}{$\begin{array}{l}\text { Recessive dystrophic epidermolysis } \\
\text { bullosa (RDEB) }\end{array}$} & COLTA1 & c.189delG (at exon 2) & HDR (IDLV donor) $)^{54}$ \\
\hline & & c.6527insC (at exon 80) & Large deletion using dual sgRNAs ${ }^{25}$ \\
\hline & & c.553 C > T (p.R185X) or c.1573 C > T (p.R525X) & $A B E^{89}$ \\
\hline \multirow[t]{3}{*}{ Sickle cell disease (SCD) } & $H B B$ & $c .20 \mathrm{~A}>\mathrm{T}$ (p.E6V or p.E7V) & HDR (IDLV donor) $)^{28}$ \\
\hline & & & HDR (AAV donor) $)^{27,29}$ \\
\hline & & & $\mathrm{PE}^{96}$ \\
\hline Tay-Sachs disease & HEXA & c.1274_1278dup & $P E^{96}$ \\
\hline $\begin{array}{l}\text { Xeroderma pigmentosum, } \\
\text { complementation group C (XPC) }\end{array}$ & $X P C$ & c.1840C > T (p.R579X) & ABE (CRISPR-Pass) ${ }^{91}$ \\
\hline
\end{tabular}

$A d v$ adenoviral vector, IDLV integrase-defective lentiviral vector, AAV adeno-associated viral vector, PTC premature termination codon.

anducing mutations that alleviate disease symptoms.

${ }^{b}$ Using the normal allele on the homologous chromosome as a template.

transposase subunits for cargo gene insertion into the target site. INTEGRATE differs from ShCAST in that it additionally requires the Tn7 transposition protein tnsA and can insert the cargo gene into the target site bidirectionally. Although both ShCAST and INTEGRATE have been tested only in Escherichia coli to date ${ }^{94,95}$, it is expected that they can be employed as great alternatives to NHEJ for gene knockin therapy through subsequent improvement, which will enable them to function in eukaryotic cells.

Another new approach involves the use of a prime editor (PE), which is composed of a reverse transcriptase (RT) and a Cas9 nickase, and a unique "prime editing" guide RNA (pegRNA) ${ }^{96}$ (Fig. 4). A PE that is recruited to the target site by a pegRNA generates a nick in the PAMcontaining strand. Because the pegRNA has a template sequence at its 3 ' end, the RT enzyme of a PE can copy the information from the template to the 3 ' end of the nicked strand of DNA. This in situ synthesized donor-templated repair of DNA enables PEs to precisely induce substitutions, including transversion and transition, as well as indels, which cannot be achieved by conventional base editors $^{71-73,96}$. In addition, PEs showed low off-target editing effects and generated negligible byproduct mutations, suggesting that PEs shows promise in medical applications $^{96}$. Indeed, PEs effectively corrected the mutations in the $H B B$ gene and HEXA gene in HEK293T cells, which cause sickle cell disease and TaySachs disease, respectively ${ }^{96}$. Furthermore, a recent study revealed that a plant prime editor (PPE), an optimized version of PEs for plant cells, can induce various substitutions and indels efficiently in rice and wheat protoplasts $^{97}$, suggesting that PEs can be applied to diverse systems, including plants and animals.

\section{Conclusion}

In this review, we have focused on CRISPR nucleasemediated gene recovery strategies rather than methods for 
gene knockout or gene regulation such as gene inhibition (CRISPRi) or activation (CRISPRa) ${ }^{98}$. Gene correction technologies have been rapidly developed and widely tested for the treatment of many genetic diseases (Table 1). Gene correction based on DNA cleavage in the absence of donor DNA is relatively simple if frameshifting will lead to gene recovery but is of limited value for correcting many types of mutations, such as substitutions. For such purposes, HDR-mediated gene correction is the most precise and well characterized method, but its usefulness is limited because of its low efficiency, lack of activity in non-dividing cells, and need for donor DNA. Although NHEJ- or MMEJ-mediated gene correction enable gene knockin even in non-dividing cells with higher efficacy than HDR, these methods still require donor DNA and are typically less precise than HDR. Furthermore, recent studies have reported unexpected outcomes, including large deletions and chromosomal translocations, after DNA cleavage ${ }^{69,70}$; such results warn against the use of DNA cleavage-mediated gene recovery strategies.

On the other hand, CRISPR-based base editors, which enable precise, highly efficient base conversion without requiring donor DNA or producing DNA cleavage, represent an alternative approach. However, CBEs and ABEs can convert bystander bases positioned within the editing window as well as target bases, generating unwanted mutations ${ }^{74,75}$ and, in such cases, failing to produce exact gene corrections. Furthermore, these tools are limited in the types of targeted base conversions they induce $(C \cdot G \rightarrow T \cdot A$ or $A \cdot T \rightarrow G \cdot C$ only). In addition, several recent studies reported genome-wide off-target deaminase effects on DNA (for CBEs) ${ }^{99,100}$ and RNA (for CBEs and $A B E s)^{101,102}$ and unwanted effects on DNA cytosines (for $\mathrm{ABEs})^{103}$, necessitating further improvement. The recently developed CAST and INTEGRATE methods represent alternative gene knockin strategies, but their use has not yet been demonstrated in mammalian cells. PE is likewise a potentially attractive tool that requires further characterization.

In summary, all gene-editing tools are associated with some limitations or side effects, such as off-target editing, but intensive research has led to rapid improvement to compensate for these limitations. In the near future, we anticipate that several genetic diseases that lack reliable drugs will be treatable with an appropriate gene recovery strategy on the basis of the underlying genomic abnormality. Such precise endogenous gene recovery will herald a new era in human genetic medicine.

\section{Acknowledgements}

This research was supported by grants from the National Research Foundation of Korea (NRF) (no. 2018M3A9H3022412), the Next Generation BioGreen 21 Program (PJ01319301), the Korea Healthcare Technology R\&D Project

(HI16C1012), and the Technology Innovation Program (no. 20000158) to S.B.
Conflict of interest

The authors declare that they have no conflict of interest.

Publisher's note

Springer Nature remains neutral with regard to jurisdictional claims in published maps and institutional affiliations.

Received: 7 April 2020 Accepted: 21 May 2020.

Published online: 10 July 2020

\section{References}

1. Kotterman, M. A., Chalberg, T. W. \& Schaffer, D. V. Viral vectors for gene therapy: translational and clinical outlook. Annu. Rev. Biomed. Eng. 17, 63-89 (2015).

2. Anguela, X. M. \& High, K. A. Entering the modern era of gene therapy. Annu Rev. Med. 70, 273-288 (2019)

3. Kim, Y. G., Cha, J. \& Chandrasegaran, S. Hybrid restriction enzymes: zinc finger fusions to Fok I cleavage domain. Proc. Natl Acad. Sci. USA 93, 1156-1160 (1996).

4. Christian, M. et al. Targeting DNA double-strand breaks with TAL effector nucleases. Genetics 186, 757-761 (2010).

5. Cong, L. et al. Multiplex genome engineering using CRISPR/Cas systems. Science 339, 819-823 (2013).

6. Mali, P. et al. RNA-guided human genome engineering via Cas9. Science $\mathbf{3 3 9}$ 823-826 (2013).

7. Cho, S. W., Kim, S., Kim, J. M. \& Kim, J.-S. Targeted genome engineering in human cells with the Cas9 RNA-guided endonuclease. Nat. Biotechnol. 31 230-232 (2013).

8. Jinek, M. et al. A programmable dual-RNA-guided DNA endonuclease in adaptive bacterial immunity. Science 337, 816-821 (2012).

9. Zetsche, B. et al. Cpf1 is a single RNA-guided endonuclease of a class 2 CRISPR-Cas system. Cell 163, 759-771 (2015).

10. Yeh, C. D., Richardson, C. D. \& Corn, J. E. Advances in genome editing through control of DNA repair pathways. Nat. Cell Biol. 21, 1468-1478 (2019).

11. Davis, A. J. \& Chen, D. J. DNA double strand break repair via nonhomologous end-joining. Transl. Cancer Res. 2, 130-143 (2013).

12. Guirouilh-Barbat, J. et al. Impact of the KU80 pathway on NHEJinduced genome rearrangements in mammalian cells. Mol. Cell 14, 611-623 (2004).

13. Li, H. L. et al. Precise correction of the dystrophin gene in duchenne muscular dystrophy patient induced pluripotent stem cells by TALEN and CRISPR-Cas9. Stem cell Rep. 4, 143-154 (2015).

14. Min, Y.-L. et al. CRISPR-Cas9 corrects Duchenne muscular dystrophy exon 44 deletion mutations in mice and human cells. Sci. Adv. 5, eaav4324 (2019).

15. Long, C. et al. Correction of diverse muscular dystrophy mutations in human engineered heart muscle by single-site genome editing. Sci. Adv. 4, eaap9004 (2018).

16. $\mathrm{Xu}$, S. et al. Editing aberrant splice sites efficiently restores $\beta$-globin expression in $\beta$-thalassemia. Blood 133, 2255-2262 (2019).

17. Iyer, $\mathrm{S}$. et al. Precise therapeutic gene correction by a simple nucleaseinduced double-stranded break. Nature 568, 561-565 (2019).

18. Bae, S., Kweon, J., Kim, H. S. \& Kim, J.-S. Microhomology-based choice of Cas9 nuclease target sites. Nat. Methods 11, 705-706 (2014).

19. Shen, M. W. et al. Predictable and precise template-free CRISPR editing of pathogenic variants. Nature $\mathbf{5 6 3}, 646-651$ (2018).

20. Kim, H. K. et al. SpCas9 activity prediction by DeepSpCas9, a deep learningbased model with high generalization performance. Sci. Adv. 5, eaax9249 (2019).

21. Kim, H. K. et al. Deep learning improves prediction of CRISPR-Cpf1 guide RNA activity. Nat. Biotechnol. 36, 239-241 (2018).

22. Dastidar, S. et al. Efficient CRISPR/Cas9-mediated editing of trinucleotide repeat expansion in myotonic dystrophy patient-derived IPS and myogenic cells. Nucleic Acids Res. 46, 8275-8298 (2018).

23. Lo Scrudato, M. et al. Genome editing of expanded CTG repeats within the human DMPK gene reduces nuclear RNA foci in the muscle of DM1 mice. Mol. Ther. 27, 1372-1388 (2019). 
24. $\mathrm{Xu}$, L. et al. CRISPR-mediated genome editing restores dystrophin expression and function in mdx mice. Mol. Ther. 24, 564-569 (2016).

25. Bonafont, J. et al. Clinically relevant correction of recessive dystrophic epidermolysis bullosa by dual sgRNA CRISPR/Cas9-mediated gene editing. Mol. Ther. 27, 986-998 (2019).

26. Park, C. Y. et al. Functional correction of large factor VIII gene chromosomal inversions in Hemophilia A patient-derived iPSCs using CRISPR-Cas9. Cell Stem Cell 17, 213-220 (2015).

27. Dever, D. P. et al. CRISPR/Cas9 $\beta$-globin gene targeting in human haematopoietic stem cells. Nature 539, 384-389 (2016).

28. Hoban, M. D. et al. CRISPR/Cas9-mediated correction of the sickle mutation in human CD34+ cells. Mol. Ther. 24, 1561-1569 (2016).

29. Martin, R. M. et al. Highly efficient and marker-free genome editing of human pluripotent stem cells by CRISPR-Cas9 RNP and AAV6 donor-mediated homologous recombination. Cell Stem Cell 24, 821-828 (2019).

30. Yang, $Y$. et al. Naïve induced pluripotent stem cells generated from $\beta$-thalassemia fibroblasts allow efficient Gene correction with CRISPR/Cas9. Stem Cells Transl. Med. 5, 267 (2016).

31. Xu, P. et al. Both TALENs and CRISPR/Cas9 directly target the HBB IVS2-654 (C > T) mutation in $\beta$-thalassemia-derived iPSCs. Sci. Rep. $\mathbf{5}$ 12065 (2015).

32. Tang, L. et al. CRISPR/Cas9-mediated gene editing in human zygotes using Cas9 protein. Mol. Genet. Genomics 292, 525-533 (2017).

33. Niu, X. et al. Combining single strand oligodeoxynucleotides and CRISPR/ Cas9 to correct gene mutations in $\beta$-thalassemia-induced pluripotent stem cells. J. Biol. Chem. 291, 16576-16585 (2016).

34. Xie, F. et al. Seamless gene correction of $\beta$-thalassemia mutations in patientspecific iPSCs using CRISPR/Cas9 and piggyBac. Genome Res. 24, 1526-1533 (2014).

35. Gao, J. et al. Viral vector-based delivery of CRISPR/Cas9 and donor DNA for homology-directed repair in an in vitro model for canine Hemophilia B. Mol. Ther. Nucleic Acids 14, 364-376 (2019).

36. Stephens, C. J. et al. Long-term correction of hemophilia B using adenoviral delivery of CRISPR/Cas9. J. Control Release 298, 128-141 (2019).

37. Guan, Y. et al. CRISPR/Cas9-mediated somatic correction of a novel coagulator factor IX gene mutation ameliorates hemophilia in mouse. EMBO Mol. Med. 8, 477-488 (2016).

38. Sung, J. J., Park, C.-Y., Leem, J. W., Cho, M. S. \& Kim, D.-W. Restoration of FVII expression by targeted gene insertion in the FVIII locus in hemophilia A patient-derived iPSCs. Exp. Mol. Med. 51, 1-9 (2019).

39. Zhu, P. et al. CRISPR/Cas9-mediated genome editing corrects dystrophin mutation in skeletal muscle stem cells in a mouse model of muscle dystrophy. Mol. Ther. Nucleic Acids 7, 31-41 (2017).

40. Long, C. et al. Prevention of muscular dystrophy in mice by CRISPR/Cas9mediated editing of germline DNA. Science 345, 1184-1188 (2014).

41. Ryu, S.M., Hur, J. W. \& Kim, K. Evolution of CRISPR towards accurate and efficient mammal genome engineering. BMB Rep. 52, 475-481 (2019).

42. Renaud, J-B. et al. Improved genome editing efficiency and flexibility using modified oligonucleotides with TALEN and CRISPR-Cas9 nucleases. Cell Rep. 14, 2263-2272 (2016)

43. $\mathrm{Wu}, \mathrm{Y}$. et al. Correction of a genetic disease in mouse via Use of CRISPR-Cas9. Cell Stem Cell 13, 659-662 (2013).

44. Zhao, L. et al. A 1-bp deletion in the $\mathrm{YC}$-crystallin leads to dominant cataracts in mice. Mamm. Genome 21, 361-369 (2010).

45. Miao, K. et al. Optimizing CRISPR/Cas9 technology for precise correction of the Fgfr3-G374R mutation in achondroplasia in mice. J. Biol. Chem. 294, 1142-1151 (2019).

46. Ortiz-Virumbrales, M. et al. CRISPR/Cas9-Correctable mutation-related molecular and physiological phenotypes in iPSC-derived Alzheimer's PSEN2 (N1411) neurons. Acta Neuropathol. Commun. 5, 77 (2017).

47. $\mathrm{Wu}, \mathrm{W}$. H. et al. CRISPR repair reveals causative mutation in a preclinical model of retinitis pigmentosa. Mol. Ther. 24, 1388-1394 (2016).

48. Park, S. H. et al. Highly efficient editing of the $\beta$-globin gene in patientderived hematopoietic stem and progenitor cells to treat sickle cell disease. Nucleic Acids Res. 47, 7955-7972 (2019).

49. van de Vrugt, H. J. et al. Effective CRISPR/Cas9-mediated correction of a Fanconi anemia defect by error-prone end joining or templated repair. Sci. Rep. 9, 768 (2019).

50. Schwank, G. et al. Functional repair of CFTR by CRISPR/Cas9 in intestinal stem cell organoids of cystic fibrosis patients. Cell Stem Cell 13, 653-658 (2013).
51. Deng, W. L. et al. Gene correction reverses ciliopathy and photoreceptor loss in iPSC-derived retinal organoids from retinitis pigmentosa patients. Stem Cell Rep. 10, 1267-1281 (2018).

52. Kocher, T. et al. Cut and paste: efficient homology-directed repair of a dominant negative KRT14 mutation via CRISPR/Cas9 nickases. Mol. Ther. 25, 2585-2598 (2017)

53. Yao, X. et al. Tild-CRISPR allows for efficient and precise gene knockin in mouse and human cells. Dev. Cell 45, 526-536 (2018).

54. Izmiryan, A. et al. Ex vivo COL7A1 correction for recessive dystrophic epidermolysis bullosa using CRISPR/Cas9 and homology-directed repair. Mol. Ther. Nucleic Acids 12, 554-567 (2018).

55. Bak, R. O., Dever, D. P. \& Porteus, M. H. CRISPR/Cas9 genome editing in human hematopoietic stem cells. Nat. Protoc. 13, 358 (2018).

56. Vakulskas, C. A. et al. A high-fidelity Cas9 mutant delivered as a ribonucleoprotein complex enables efficient gene editing in human hematopoietic stem and progenitor cells. Nat. Med. 24, 1216-1224 (2018).

57. Hustedt, N. \& Durocher, D. The control of DNA repair by the cell cycle. Nat. Cell Biol. 19, 1-9 (2017)

58. He, X. et al. Knock-in of large reporter genes in human cells via CRISPR/Cas9induced homology-dependent and independent DNA repair. Nucleic Acids Res. 44, e85-e85 (2016).

59. Suzuki, K. et al. In vivo genome editing via CRISPR/Cas9 mediated homologyindependent targeted integration. Nature 540, 144-149 (2016).

60. Suzuki, K. \& Izpisua Belmonte, J. C. In vivo genome editing via the HITI method as a tool for gene therapy. J. Hum. Genet. 63, 157-164 (2018).

61. D'Cruz, P. M. et al. Mutation of the receptor tyrosine kinase gene Mertk in the retinal dystrophic RCS rat. Hum. Mol. Genet. 9, 645-651 (2000).

62. Nakade, S. et al. Microhomology-mediated end-joining-dependent integration of donor DNA in cells and animals using TALENs and CRISPR/Cas9. Nat Commun. 5, 5560 (2014).

63. Yao, X. et al. CRISPR/Cas9-mediated precise targeted integration in vivo using a double cut donor with short homology arms. EBioMedicine 20, 19-26 (2017).

64. Yao, X. et al. Homology-mediated end joining-based targeted integration using CRISPR/Cas9. Cell Res. 27, 801-814 (2017).

65. Yao, X. et al. CRISPR/Cas9-mediated targeted integration in vivo using a homology-mediated end joining-based strategy. J. Vis. Exp. 133, 56844 (2018).

66. Rees, H. A. \& Liu, D. R. Base editing: precision chemistry on the genome and transcriptome of living cells. Nat. Rev. Genet. 19, 770-788 (2018).

67. Haapaniemi, E., Botla, S., Persson, J., Schmierer, B. \& Taipale, J. CRISPR-Cas9 genome editing induces a p53-mediated DNA damage response. Nat. Med. 24, 927-930 (2018)

68. Ihry, R. J. et al. p53 inhibits CRISPR-Cas9 engineering in human pluripotent stem cells. Nat. Med. 24, 939-946 (2018).

69. Kosicki, M., Tomberg, K. \& Bradley, A. Repair of double-strand breaks induced by CRISPR-Cas9 leads to large deletions and complex rearrangements. Nat. Biotechnol. 36, 765-771 (2018).

70. Shin, H. Y. et al. CRISPR/Cas9 targeting events cause complex deletions and insertions at 17 sites in the mouse genome. Nat. Commun. 8 15464 (2017).

71. Komor, A. C., Kim, Y. B., Packer, M. S., Zuris, J. A. \& Liu, D. R. Programmable editing of a target base in genomic DNA without double-stranded DNA cleavage. Nature 533, 420-424 (2016).

72. Gaudelli, N. M. et al. Programmable base editing of A.T to G.C in genomic DNA without DNA cleavage. Nature 551, 464-471 (2017).

73. Nishida, K. et al. Targeted nucleotide editing using hybrid prokaryotic and vertebrate adaptive immune systems. Science $\mathbf{3 5 3}$, aaf8729. https://doi.org/ 10.1126/science.aaf8729 (2016).

74. Tan, J., Zhang, F., Karcher, D. \& Bock, R. Engineering of high-precision base editors for site-specific single nucleotide replacement. Nat. Commun. 10, 439 (2019).

75. Kim, Y. B. et al. Increasing the genome-targeting scope and precision of base editing with engineered Cas9-cytidine deaminase fusions. Nat. Biotechnol. 35 371-376 (2017)

76. Zafra, M. P. et al. Optimized base editors enable efficient editing in cells, organoids and mice. Nat. Biotechnol. 36, 888-893 (2018).

77. Komor, A. C. et al. Improved base excision repair inhibition and bacteriophage Mu Gam protein yields C:G-to-T:A base editors with higher efficiency and product purity. Sci. Adv. 3, eaao4774 (2017). 
78. Koblan, L. W. et al. Improving cytidine and adenine base editors by expression optimization and ancestral reconstruction. Nat. Biotechnol. 36 843-846 (2018).

79. Lim, C. K. W. et al. Treatment of a mouse model of ALS by in vivo base editing. Mol. Ther. https://doi.org/10.1016/j.ymthe.2020.01.005 (2020).

80. Liang, P. et al. Correction of $\beta$-thalassemia mutant by base editor in human embryos. Protein Cell 8, 811-822 (2017).

81. Arbustini, E. et al. Identification of sixty-two novel and twelve known FBN1 mutations in eighty-one unrelated probands with Marfan syndrome and other fibrillinopathies. Hum. Mutat. 26, 494-494 (2005).

82. Zeng, Y. et al. Correction of the marfan syndrome pathogenic FBN1 mutation by base editing in human cells and heterozygous embryos. Mol. Ther. 26, 2631-2637 (2018)

83. Villiger, L. et al. Treatment of a metabolic liver disease by in vivo genome base editing in adult mice. Nat. Med. 24, 1519-1525 (2018).

84. Huang, T. P. et al. Circularly permuted and PAM-modified Cas9 variants broaden the targeting scope of base editors. Nat. Biotechnol. 37, 626-631 (2019)

85. Ryu, S.-M. et al. Adenine base editing in mouse embryos and an adult mouse model of Duchenne muscular dystrophy. Nat. Biotechnol. 36 536-539 (2018).

86. Song, C.-Q. et al. Adenine base editing in an adult mouse model of tyrosinaemia. Nat. Biomed. Eng. 4, 125-130 (2020).

87. Mittapalli, V. R. et al. Injury-driven stiffening of the dermis expedites skin carcinoma progression. Cancer Res. 76, 940-951 (2016).

88. Rashidghamat, E. \& McGrath, J. A. Novel and emerging therapies in the treatment of recessive dystrophic epidermolysis bullosa. Intractable Rare Dis. Res. 6, 6-20 (2017).

89. Osborn, M. J. et al. Base editor correction of COL7A1 in recessive dystrophic epidermolysis bullosa patient-derived fibroblasts and iPSCs. J. Invest. Dermatol. 140, 338-347 (2020). e335.
90. Li, X. et al. Programmable base editing of mutated TERT promoter inhibits brain tumour growth. Nat. Cell Biol. 22, 282-288 (2020).

91. Lee, C. et al. CRISPR-Pass: gene rescue of nonsense mutations using adenine base editors. Mol. Ther. 27, 1364-1371 (2019).

92. Peters, J. E., Makarova, K. S., Shmakov, S. \& Koonin, E. V. Recruitment of CRISPRCas systems by Tn7-like transposons. Proc. Natl Acad. Sci. USA 114 E7358-e7366 (2017)

93. Faure, G. et al. CRISPR-Cas in mobile genetic elements: counter-defence and beyond. Nat. Rev. Microbiol. 17, 513-525 (2019).

94. Strecker, J. et al. RNA-guided DNA insertion with CRISPR-associated transposases. Science 365, 48-53 (2019).

95. Klompe, S. E., Vo, P. L. H., Halpin-Healy, T. S. \& Sternberg, S. H. Transposonencoded CRISPR-Cas systems direct RNA-guided DNA integration. Nature 571, 219-225 (2019).

96. Anzalone, A. V. et al. Search-and-replace genome editing without doublestrand breaks or donor DNA. Nature 576, 149-157 (2019).

97. Lin, Q. et al. Prime genome editing in rice and wheat. Nat. Biotechnol. 38 582-585 (2020).

98. Pickar-Oliver, A. \& Gersbach, C. A. The next generation of CRISPR-Cas technologies and applications. Nat. Rev. Mol. Cell Biol. 20, 490-507 (2019).

99. Zuo, E. et al. Cytosine base editor generates substantial off-target singlenucleotide variants in mouse embryos. Science 364, 289-292 (2019).

100. Jin, S. et al. Cytosine, but not adenine, base editors induce genome-wide offtarget mutations in rice. Science 364, 292-295 (2019).

101. Grünewald, J. et al. Transcriptome-wide off-target RNA editing induced by CRISPR-guided DNA base editors. Nature 569, 433-437 (2019).

102. Zhou, C. et al. Off-target RNA mutation induced by DNA base editing and its elimination by mutagenesis. Nature 571, 275-278 (2019).

103. Kim, H. S., Jeong, Y. K., Hur, J. K., Kim, J.-S. \& Bae, S. Adenine base editors catalyze cytosine conversions in human cells. Nat. Biotechnol. 37, 1145-1148 (2019) 